\title{
Short Bowel syndrome
}

\author{
Professor KL Sampath Kumar, ${ }^{1}$ Dr P Krishna Murthy ${ }^{2}$ \\ ${ }^{\text {I}}$ Professor KL Sampath Kumar \\ ${ }_{,}^{2}$ Professor of General surgery,Narayana Medical College, Chinthareddy Palem,NELLORE \\ Andhra Pradesh
}

\section{Introduction}

Small bowel plays an important role in the digestion and absorption of various nutrients from the enteral diet. The small intestine consists of three parts, duodenum, jejunum and ileum. Apart from duodenal length of $25 \mathrm{~cm}$, the rest of the small intestine length varies between four and six meters. Though the majority of the digestion takes place in the proximal bowel, the distal bowel, especially the terminal part of ileum plays a very significant role in the digestion and absorption of fats and in entero hepatic circulation of bile salts. The ileum is guarded by an ileocaecal valve, which is an important cog in the wheel of the intestinal integrity and its function. If for any reason, the length of the functioning bowel is shortened either as a result of surgery or any disease, the individual invariably suffers from defective digestion and malabsorption and in some cases ends in fatality. This condition is clinically known as the short bowel syndrome.

\section{Definition}

Short bowel syndrome is defined as a type of intestinal failure which results from surgical resection for a congenital defect or an acquired disease or diseases associated with loss of absorption and is characterised by the inability to maintain protein, energy, fluid, electrolyte and micronutrients balance when on a conventionally accepted normal enteral diet (1). Generally, in adults, short bowel syndrome occurs when there is a less than 200 $\mathrm{cm}$ of functioning small bowel (2).

\section{Aetiology:}

There are many aetiological causes for short bowel syndrome. These causes differ based on age groups. In the neonates, it is mainly due to congenital volvulus neonatorum. In the infants, it is due to necrotising enterocolitis. In the children, short bowel syndrome occurs as a result of intestinal resection for trauma or malignant conditions. However, in adults, there are many causes like mesenteric ischaemia, inflammatory bowel diseases, intestinal malignancies and radiation necrosis (2), (3), (4). In this $21^{\text {st }}$ century, gunshot wounds or penetrating injuries of the abdomen resulting in mesenteric tear or extensive small bowel injury requiring extensive small bowel resection also result in short bowel syndrome.

It is important to differentiate between true short bowel syndrome and intestinal failure producing the clinical features of short bowel syndrome. Usually, the intestinal failure refers to a condition which results from intestinal dysmotility disorder or diseases associated with significant loss of intestinal primary function of digestion and absorption (1).

There is an increasing incidence of short bowel syndrome in the present society in the last few decades. It is of multifactorial in origin. Earlier, most of the patients succumb to the primary disease itself. But, nowadays, due to advancements in the diagnostic procedures, improved operative techniques and excellent postoperative management with respect to sepsis and resuscitative measures, many of these patients are able to survive only to suffer from short bowel syndrome. In addition to the above factors, one more important factor is easy availability of total parenteral nutrition for these patients who suffer from acute intestinal failure. Though the Indian statistics are not available, the world wide references indicate that the incidence of short bowel syndrome patients, who requires definitive management, is progressively increasing (5), (6), (7).

\section{Patho physiology:}

The small intestine has a high degree of adaptive capacity whenever there is a significant reduction in the available length of functioning small intestine for normal digestion and absorption (8), (9), (10).The post intestinal resection adaptation takes place over a period of about 18 months to 24 months. It is divided into three phases.

1. Acute phase.

2. Adaptive phase.

3. Maintenance phase. 


\section{Acute Phase:}

This acute phase starts immediately after the surgical resection and lasts for about four weeks to twelve weeks. This phase is characterised by diarrhoea, malabsorption, intestinal dysmotility and gastric hyper secretion. Intestinal resection compromises the endocrinal capacity of the gut. Cholecysto kinin, secretin, gastric inhibitory poly peptide which are normally produced in the intestinal lumen by the intestinal mucosal cells are reduced resulting in a faster gastric emptying, hyper gastrinaemia, inactivation of digestive enzymes, loss of bile salts and rapid intestinal transit.

\section{Adaptation Phase:}

Once the patient is stabilised, intestinal adaptation phase starts, as early as the third day and may continue for 18 months. The structural changes include an increase in the length and diameter of the intestinal remnant. The thickness of the intestinal wall increases. On microscopic examination, one can find the evidence of hypertrophy of intestinal villi, an increase in the number of micro villi, an increase in the number of intestinal crypts and an increase in the depth of the intestinal crypts. In addition, there is an increase in the differentiation of specialised mucosal cells and angiogenesis (11) (12) (13). All these structural changes help in increasing the surface area for digestion and absorption. In addition, there is a decrease in the intestinal peristaltic activity thus increasing the intestinal transit time so that there is adequate time for the digestion and absorption. It has been noticed by various studies that there is an increase in the number of carrier proteins and their intrinsic activity.(14). It has been noticed if the patient is started on an enteral diet at the earliest, intestinal adaptation is faster as it results in intestinal luminal stimulation (15).

\section{Maintenance Phase:}

Once the patient recovers and the adaptation phase is over, the intestine reaches the maintenance phase. Here, though no fresh anatomical or functional changes occur, the intestine continues to show the changes which have taken place during the adaptation phase. However, during this phase, the patient needs proper evaluation so as to decide the requirement of any specific nutritional requirement and the same to be replenished as and when needed so that the quality of life is not affected.

\section{Clinical Features:}

The clinical features of short bowel syndrome depend on many factors. The important factors are as follows:

(A). the length of functioning intestinal remnant.

(B). the specific part of the lost bowel

(C). the presence or absence of ileo caecal valve.

(D). the presence or absence of colon.

(E). the quality of the intestinal adaptation.

The short bowel results in accumulation of osmotically active particles in the small bowel retaining more water with in the intestinal lumen resulting in diarrhoea. The loss of small bowel length reduces pancreatic and biliary secretions and an increased gastric secretion lowers the alkalinity of the intestinal lumen. These factors impair the action of digestion. Loss of terminal ileum results in loss of bile salts and impairment of digestion and absorption of fats resulting in steotorrhoea. A loss of ileo caecal valve decreases the intestinal transit time and the absorption is impaired. In addition the loss of ileo caecal valve results in colonic bacterial colonisation of small bowel lumen aggravating diarrhoea and malnutrition.

The following observations are made after various studies regarding the clinical features of short bowel syndrome (16) (17).

1. The clinical features of nutritional deficiency of short bowel syndrome are variable.

2. Loss of proximal bowel is well tolerated than the distal bowel because the distal bowel is able to get adapted faster and better.

3. Loss of the terminal ileum with its ileo caecal valve is more detrimental to the patient.

4. The presence of colon helps in the intestinal adaptation by substantially increasing fluid retention capability and the colonic bacteria is able to digest carbohydrates into absorbable short chain fatty acids.

5. Jejuna resection results in minimal clinical abnormality.

\section{Investigations}

Short bowel syndrome is a clinical diagnosis based on the history and the clinical findings of various nutritional deficiencies. Most of the investigations are directed towards the assessment of the extent of malnutrition and specific nutritional deficiency. However, the circulating plasma levels of Citrulline may provide the information regarding the available length of functioning small bowel in a patient suffering from short bowel syndrome. Citrulline is a non protein amino acid produced by the intestinal mucosa. Normal value is 
more than 40 micro moles per litre. In short bowel syndrome patients, the plasma Citrulline levels are reduced and these levels are well correlated to the available bowel lengths.

\section{Treatment:}

All short bowel patients are treated by a multidisciplinary team of various specialists in order to enhance intestinal recovery after correcting the specific nutritional deficiencies. Once the patient is successfully resuscitated and stabilised and the patient is free from sepsis, the treatment options are nutritional support and pharmacological treatment with nutritional support. And occasionally the surgical treatment is offered.

\section{Dietary Support:}

Ideally, the patients are given parenteral nutrition for the required time only and the enteral diet is started at the earliest once the patient is able to tolerate oral nutrition because the oral diet stimulates the intestinal mucosal cells to function effectively (18). It is proved that the patient is benefited if the patient is given small meals, six to eight times a day rather than two or three big meals (19). Whenever ileal resection is greater than $100 \mathrm{~cm}$, diet should contain less of oxalates but rich in calcium to reduce the risk of formation of calcium oxalate stones in the urinary tract. The diet should be rich in soluble fibre and carbohydrates, so as to increase the intestinal transit time, so that effective absorption takes place. Moreover, if the colon is preserved, the colonic bacteria can act on the carbohydrates and form easily absorbable short chain fatty acids. It is advisable to administer the elemental diet. The elemental diets are cheaper, less hyperosmolar, improve intestinal adaptation and are well tolerated. The nutritional substances are also easily digested and easily absorbed. In general, 40 to $50 \%$ of dietary calories should come from complex carbohydrates and 30 to $40 \%$ from lipids.

\section{Pharmacological Support:}

There are different types of medicines which are found to be beneficial in short bowel syndrome patients. Loperamide or Diphenoxylate with atropine may help in diminishing intestinal motility. Intestinal sedatives like Codeine and opium which act centrally may also help in reducing the intestinal motility. Once the intestinal motility is reduced, there is an increase in the intestinal transit time so that there is adequate time for the digestion and absorption of nutrients from the diet. These drugs are administered preferentially 30 to 60 minutes prior to meals to ensure greater efficiency. The patients of short bowel syndrome show increased gastric secretions thus impairing digestion. So, proton pump inhibitors and H-2 receptor blockers can be administered to these patients. If there is not much of improvement, one can try Alpha- 2 adrenergic agonists. Probiotics and probiotics when administered orally, the patients showed increased intestinal adaptation, reduced bacterial translocation and restored the intestinal bacterial flora thus enhancing the health and function of the intestinal mucosa. These are more effective in the paediatric age group of patients rather than in adults (20). Glucagon like Peptide-2 is a peptide normally produced by enterocytes on intestinal stimulation to maintain growth and maintenance of intestinal epithelium. It helps in the absorption of nutrients, inhibits intestinal motility and gastric hyper secretion. This peptide is synthetically produced as Teduglutide.Somatostatin analogue, somatotropin helps in the intestinal adaptation by an increase in the size of intestinal villi, the number of intestinal villi and intestinal crypts. It also helps in the increase of the depth of intestinal crypts (21)

\section{Surgical Treatment:}

Surgery plays an important role in preventing, helping or even reversing intestinal failure in some cases if not in all cases. One should practice "Relook laparotomy" in deciding about the resection of doubtful viable bowel in cases of mesenteric vessel catastrophe and abdominal injuries. Methods to increase the intestinal transit time by creating artificial sphincters and valves did not yield good results. Similarly, retrograde intestinal pacing with electrodes also did not show improvement. Some surgeons tried to reverse 10 to $15 \mathrm{~cm}$ segments of bowel to increase intestinal transit time, but the results were not encouraging. Some surgeons tried to increase the length of the small intestine by colonic interposition, but did not get good results.The dilatation of the remnant of the small intestine occurs as an adaptation mechanism in patients suffering from short bowel syndrome. Surgeons took advantage of this situation and two surgical methods are introduced to increase the length of the small bowel.

1. Bianchi Procedure: A $5 \mathrm{~cm}$ longitudinal avascular plane is created along the mesenteric side of the dilated loop of the intestine. Then the dilated loop is longitudinally divided and each half is then tubularised forming two narrow loops to be anastomosed end to end in an isoperistaltic mode. Thus this procedure results in intestinal loop with half of its diameter but with twice its length (22) (23).

2. Serial Transverse Enteroplasty (STEP): Here, the dilated intestinal loop is narrowed by applying metallic clamps perpendicular to the longitudinal axis of the intestine in a zigzag pattern. This procedure results in an increase in the length and a decrease in the diameter of the intestine (24). 


\section{Small Bowel Transplantation:}

Small bowel transplantation is life saving option for short bowel syndrome patients. Though it was done in the last century itself, the results were disastrous. After the introduction of effective immune suppressive agents like Tacrolimus, the results are improving. In an isolated intestinal transplantation, the arterial connection is established through anastomosis of the donor superior mesenteric artery with the recipient infra renal aorta. The venous drainage is achieved by anastomosis of the donor superior mesenteric vein to the recipient portal vein or inferior vena cava. Bowel continuity is established through anastomosis of the proximal jejunal graft to the recipient distal duodenum and the distal ileum is brought out as an ileostomy to allow monitoring of the graft. Sepsis and graft rejection are common complications.

\section{Conclusion}

Short bowel syndrome is a condition with a greater variability both in aetiology and in its clinical manifestations. Over the years, various treatment options are developed to improve the quality of life and survival of these patients. If nutritional supplementation cannot support the life adequately, surgical treatment may be required including intestinal transplantation.

\section{References}

[1]. O' Keefe SJ, Buchnan AL, Fishbean TA, Jeebhoy KN, Jepperson PB, Shalfer J. Short bowel syndrome and intestinal failure: Consensus definition and over view. Clin Gastroenterol Hepatol. 2006; 4: 6-10.

[2]. Buchman AL. Etiology and initial management of short bowel syndrome. Gastroenterology. 2006; 130: 505-515.

[3]. Parrish CR. The clinical guide to short bowel syndrome. Pract Gastroenterol. 2005; 29: 67-106.

[4]. Nightingale JMD. Management of patients with a short bowel syndrome. World J Gastroenterol. 2001; 7: 742-751.

[5]. Lennard-Jones JE. Indications and need for long term parenteral nutrition: implications for intestinal transplantation. Transplant Proc. 1990; 22(6): 2427-2429.

[6]. Byrne TA, Persinger RL, Young LS et al. A new treatment for patients with short bowel syndrome. Growth hormone, glutamine and a modified diet. Ann Surg. 1995; 222(3): 243-254.

[7]. Moreno JM, Planas m, Lesha M etal. (The year 2002 national register on home based parenteral nutrition). Nutr Hosp. 2005; 20(4): 249-253.

[8]. Keller J, Panter H, Layer P. Management of the short bowel syndrome after extensive small bowel resection. Best Pract Res Clin Gastroenterol. 2004; 18: 977-992.

[9]. Seetharam P, Rodrigues G. Short bowel syndrome: a review of management. Saudi J Gastroenterol. 2011; 17: $229-235$.

[10]. Tappenden KA. Pathophysiology of the short bowel syndrome: consideration of resected and residual anatomy. J parenter Enter Nutr. 2014; 38: 145-155.

[11]. Doldi SB. Intestinal adaptation following jejuno ileal bypass. Clin Nutr. 1991; 10: 138-142.

[12]. Weale AR, Edwards AG, Bailey M, Lear PA. Intestinal adaptation after massive intestinal resection. Postgrad Med.2005; 81: 178184.

[13]. Cazals-Hatlm D, Noordini ML. Morphological adaptation with preserved proliferation/ transporter content in the colon of patient with short bowel syndrome. Am J Physiol Gastrointest Liver Physiol.2009; 297: G116-G123.

[14]. Drozdowski L, Thomson AB. Intestinal mucosal adaptation. World J Gastro enterol. 2006; 12: 4614-4617.

[15]. Biner JE, Taylor RG, Justice F, Paris MC, Soureal M, Nag E et al. Influence of diet complexity on intestinal adaptation following small bowel resection in a preclinical model. J Gastro enterol Hepatol. 2002; 37: 161-170.

[16]. Walls EA. An over view of short bowel syndrome management: adherence, adaptation and practical recommendations. J Acad Nutr Diet. 2013; 113: 1200-1208.

[17]. Jepperen PB. Spectrum of short bowel syndrome in adults: intestinal insufficiency to intestinal failure. J Parenter Enteral Nutr. 2014; 38: 85-135.

[18]. Matarise LE, Steiger E. Dietary and medical management of short bowel syndrome in adult patient. J Clin Gastroenterol. 2006; 40: S 85- S 93

[19]. Campher C, Wenkles M, Boulatta JI. Nutritional management of short bowel syndrome. Clin Nutr Surg Patients. 2008 ; 2: $148-153$.

[20]. Reddy VS, Patoli SK, Rao S. Role of probiotics in short bowel syndrome in infants and children - a systematic review. Nutrients 2013; 5: 669-679.

[21]. Jepperen PB. Pharmacological options for intestinal rehabilitation in patients with short bowel syndrome. J Parenter Enter Nutr. 2014; 38: S45- S50.

[22]. Bianchi A. Longitudinal intestinal lengthening and tailoring: results in 20 children. JR Soc Med. 1997; 90: 429-432.

[23]. Kishori RI. Surgical management of short bowel syndrome. J Parenter Enter Nutr. 2014; 20: 1-7.

[24]. Kim HB, Fauza D, Garja J, Oh JI, Nurko S, Jaksia T. Serial Transverse Enteroplasty (STEP): a novel bowel lengthening procedure. J Paediatr Surg. 2003; 38: 425- 429. 